\title{
Coulomb corrections for Bose-Einstein correlations in whole momentum transfer region: Proposal of seamless fitting
}

\author{
M. Biyajima ${ }^{1 *}$ T. Mizoguchi ${ }^{2}$, T.Osada $^{3}$ and G. Wilk ${ }^{4} \dagger$ \\ ${ }^{1}$ Department of Physics, Faculty of Science, \\ Shinshu University, Matsumoto 390, Japan \\ ${ }^{2}$ Toba National College of Maritime Technology, Toba 517, Japan \\ ${ }^{3}$ Department of Physics, Tohoku University, Sendai 980, Japan \\ ${ }^{4}$ Soltan Institute for Nuclear Studies, Nuclear Theory Department \\ Hoża 69, PL-00-681 Warsaw, Poland
}

October 24, 2019

\begin{abstract}
We applied an improved Coulomb correction method developed by us recently to data on identical $K K$-pairs production in $\mathrm{S}+\mathrm{Pb}$ and $p+\mathrm{Pb}$ reactions at $200 \mathrm{GeV} / \mathrm{c}$ obtained by NA44 Collaboration. To analyse the whole range of the momentum transfers measured the method of "seamless fitting" has been proposed and used together with the asymptotic expansion formula for the Coulomb wave function. We found that such Coulomb corrections lead sometimes to different than previously reported (by NA44 Collaboration) interaction region and strongly influence the long range correlations.
\end{abstract}

Preprint DPSU-95-4 (July, 1995)

Introduction: Recently NA44 Collaboration has reported their data on the Bose-Einstein correlations (BEC) of $K^{ \pm} K^{ \pm}$pairs produced in $\mathrm{S}+\mathrm{Pb}$ and $p+\mathrm{Pb}$ reactions at $200 \mathrm{GeV} / \mathrm{c}$ [1]. In our previous work [2] we have analysed these data by making use of the various source functions with the long range correlation (without, however, invoking any sort of Coulomb corrections). The data [1] have been corrected for Coulomb interactions by applying only the Gamow factor. As was pointed out some time ago by Bowler this is, however, not sufficient [3. In our recent works [4 Coulomb corrections was presented but not yet applied to any concrete data set. In the present Letter, we apply it therefore to the analysis of data for $K^{ \pm} K^{ \pm}$pairs production in $\mathrm{S}+\mathrm{Pb}$ and $p+\mathrm{Pb}$ reactions

*e-mail: minoru44@jpnyitp.bitnet

†e-mail: Wilk@fuw.edu.pl 
mentioned above (in the whole measured momentum transfer region) and compare our results with those obtained before in [2]. To be able to analyse the whole range of measured momentum transfer $Q$ and to avoid wild oscillations developing at large $Q$ 's (cf. Fig. 1a) we have to use the the asymptotic expansion of the Coulomb wave function together with the procedure of "seamless fitting" (SF) explained below (cf. Figs. 1b and 2).

Theoretical formula of BEC with Coulomb wave function: To write down an amplitude $A_{12}$ satisfying Bose-Einstein statistics it is convenient to decompose the wave function of identical (charged in our case) bosons with momenta $p_{1}$ and $p_{2}$ into the wave function of the center-of-mass system (c.m.) with total momentum $P=\frac{1}{2}\left(p_{1}+p_{2}\right)$ and the inner wave function with relative momentum $Q=\left(p_{1}-p_{2}\right)=2 q$. It allows us to express $A_{12}$ in terms of the confluent hypergeometric function $\Phi$ []:

$$
\begin{aligned}
A_{12} & =\frac{1}{\sqrt{2}}\left[\Psi(\mathbf{q}, \mathbf{r})+\Psi_{S}(\mathbf{q}, \mathbf{r})\right], \\
\Psi(\mathbf{q}, \mathbf{r}) & =\Gamma(1+i \eta) e^{-\pi \eta / 2} e^{i \mathbf{q} \cdot \mathbf{r}} \Phi(-i \eta ; 1 ; i q r(1-\cos \theta)), \\
\Psi_{S}(\mathbf{q}, \mathbf{r}) & =\Gamma(1+i \eta) e^{-\pi \eta / 2} e^{-i \mathbf{q} \cdot \mathbf{r}} \Phi(-i \eta ; 1 ; i q r(1+\cos \theta)),
\end{aligned}
$$

where $r=x_{1}-x_{2}$ and the parameter $\eta=m \alpha / 2 q$. Assuming factorization in the source functions, $\rho\left(r_{1}, r_{2}\right)=\rho\left(r_{1}\right) \rho\left(r_{2}\right)=\rho(R) \rho(r)$ (here $R=\frac{1}{2}\left(x_{1}+x_{2}\right)$ ), one obtains the following expression for theoretical BEC formula [3] including the improved Coulomb correction [5]:

$$
\begin{aligned}
N^{( \pm \pm)} / N^{\mathrm{BG}} & =\frac{1}{G(q)} \int \rho(R) d^{3} R \int \rho(r) d^{3} r\left|A_{12}\right|^{2} \\
& =\sum_{n=0}^{\infty} \sum_{m=0}^{\infty} \frac{(-i)^{n}(i)^{m}}{n+m+1}(2 q)^{n+m} I_{R}(n, m) A_{n} A_{m}^{*} \\
& \times\left[1+\frac{n ! m !}{(n+m) !}\left(1+\frac{n}{i \eta}\right)\left(1-\frac{m}{i \eta}\right)\right] \\
& =\left(1+\Delta_{1 \mathrm{C}}\right)+\left(\Delta_{\mathrm{EC}}+E_{2 \mathrm{~B}}\right),
\end{aligned}
$$

where $G(q)=2 \pi \eta /\left(e^{2 \pi \eta}-1\right)$ denotes Gamow factor and the first and the second parentheses in eq. (3) correspond to the first and the second terms in eq. (2) 1 and

$$
I_{R}(n, m)=4 \pi \int d r r^{2+n+m} \rho(r), \quad A_{n}=\frac{\Gamma(i \eta+n)}{\Gamma(i \eta)} \frac{1}{(n !)^{2}} .
$$

To analyse data corrected only by the Gamow factor using our formulae we should use the following ratio:

$$
\begin{aligned}
N^{( \pm \pm: G C)} / N^{\mathrm{BG}}(Q=2 q) & =R_{\mathrm{CC}} / G(q) \\
& =c\left(1+\Delta_{1 \mathrm{C}}+\Delta_{\mathrm{EC}}\right)\left[1+\lambda \frac{E_{2 \mathrm{~B}}}{1+\Delta_{1 \mathrm{C}}+\Delta_{\mathrm{EC}}}\right](1+\gamma Q) .
\end{aligned}
$$

\footnotetext{
${ }^{1}$ For the exact formulae for $\Delta_{\mathrm{EC}}$ and $E_{2 \mathrm{~B}}$ see $\left[\begin{array}{l}5 \\ \text {. }\end{array}\right.$
} 
It should be noted that the normalization and an effective degree of coherence, i.e., the denominator of the ratio $E_{2 \mathrm{~B}} /\left(1+\Delta_{1 \mathrm{C}}+\Delta_{\mathrm{EC}}\right)$, are related to each other. Notice also that other parameters like the additional normalization factor $c$, the long range correlation $\gamma$ and $\lambda$ are introduced by hand.

Source function: To obtain an explicit expression, we have to decide on some form of the source function. In the present Letter, we shall use the Gaussian source distribution, $\rho(r)=\frac{\beta^{3}}{\sqrt{\pi^{3}}} \exp \left(-\beta^{2} r^{2}\right)$. For this type of source function we have the following formulae for the elements of eqs. (3) and (4) [5]:

$$
\begin{aligned}
I_{R}^{\mathrm{G}}(n, m) & =\frac{2}{\sqrt{\pi}}\left(\frac{1}{\beta}\right)^{n+m}(n+m+1) \Gamma\left(\frac{n+m+1}{2}\right), \\
E_{2 \mathrm{~B}} & =\exp \left(-\frac{q^{2}}{\beta^{2}}\right) .
\end{aligned}
$$

To analyze data corrected by the Coulomb wave function as was done in [6], we should modify the formula (4) replacing it by the following one:

$$
\begin{aligned}
N^{( \pm \pm: C C)} / N^{\mathrm{BG}}(Q=2 q) & =\frac{R_{\mathrm{CC}}}{G(q)\left(1+\Delta_{1 \mathrm{C}}+\Delta_{\mathrm{EC}}\right)} \\
& =c\left[1+\lambda \frac{E_{2 \mathrm{~B}}}{1+\Delta_{1 \mathrm{C}}+\Delta_{\mathrm{EC}}}\right](1+\gamma Q) .
\end{aligned}
$$

For the sake of reference, we write down here also the conventional formula (i.e., a kind of standard formula without corrections due to the final state interactions):

$$
N^{( \pm \pm: \text {Standard })} / N^{\mathrm{BG}}(Q=2 q)=c\left[1+\lambda E_{2 \mathrm{~B}}\right](1+\gamma Q) .
$$

Asymptotic expansion of the Coulomb wave function: First of all, it should be noted that the expansion in eq. (3) has to be the limited to $q_{\text {limit }}(=Q / 2)$ only due to mathematical properties of the confluent hypergeometric function used [7]. This can be seen as wild oscillation developing in Fig. 1 (a) where the eq. (仼) has been simply used. If we, instead, set the Coulomb correction to zero in the region $q>q_{\text {limit }}$ limit, a small step appears as seen in Fig. 1 (b). Therefore in order to analyse in a consistent way the whole region of the momentum transfer measured we have to use the following asymptotic expansion of the Coulomb wave function:

$$
\begin{aligned}
A_{12}^{\text {asym }}= & \frac{1}{\sqrt{2}}\left[\Psi^{\text {asym }}(\mathbf{q}, \mathbf{r})+\Psi_{\mathrm{S}}^{\text {asym }}(\mathbf{q}, \mathbf{r})\right] \\
\Psi^{\text {asym }}(\mathbf{q}, \mathbf{r})= & \exp \{i(q z-\eta \ln (r-z))\} \times\left(1+\frac{\eta^{2}}{i q(r-z)}\right) \\
& +f(\theta) \frac{\exp \{i(q r-\eta \ln (2 q r))\}}{r}, \\
\Psi_{\mathrm{S}}^{\text {asym }}(\mathbf{q}, \mathbf{r})= & \exp \{i(-q z-\eta \ln (r+z))\} \times\left(1+\frac{\eta^{2}}{i q(r+z)}\right) \\
& +f(\pi-\theta) \frac{\exp \{i(q r-\eta \ln (2 q r))\}}{r},
\end{aligned}
$$

where $z=r \cos \theta$ and

$$
f(\theta)=-\frac{\eta}{2 q} \frac{1}{\sin ^{2}(\theta / 2)} \exp \{-2 i \eta \ln \sin (\theta / 2)+2 i \arg \Gamma(1+i \eta)\}
$$


In analyses we should assure a smooth connection between both regions of $q=Q / 2$. To avoid the divergence of denominators $\left(1 \pm \cos \theta\right.$ ), we have to introduce a cutoff parameter $\epsilon$ (of the order of $\epsilon \simeq 10^{-3}$ ) such that $(1 \pm \cos \theta)>\epsilon$ always ?. This procedure, shown as a flow chart in fig. 2, is called the "seamless fitting (SF)".

Analyses of data by SF method: Our results obtained in terms of the new formula (14) are shown in Table I and Figs. 3 and 4. Whereas the parameter $\gamma$ (representing here the influence of long range correlations) increases now noticeably in comparison with that obtained previously in [4] we find that the interaction region represented by $R=1 / 2 \beta$ remains $\mathrm{S}+\mathrm{Pb}$ reactions almost the same. Only in $p$ $+\mathrm{Pb}$ collisions the estimated $R$ becomes larger with inclusion of Coulomb correction than that obtained

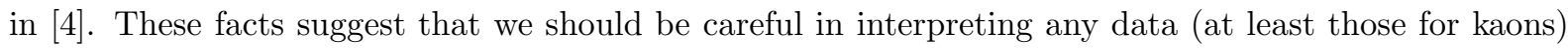
which were corrected for Coulomb interactions only by the Gamow factor.

Concluding remarks: We have proposed the possible method of applying the Coulomb correction for the BEC in the whole region of momentum transfer, the "seamless fitting" (SF). We confirm that this method works well when analysing data corrected only by the Gamow factor.

Our analyses of data of $K K$ pairs in $\mathrm{S}+\mathrm{Pb}$ reaction [1] shows (cf. Table I) that $R\left(K^{+} K^{+}\right)=4 \mathrm{fm}$ and $R\left(K^{-} K^{-}\right)=3 \mathrm{fm}$ (i.e., they differ substantially), contrary to the estimation provided in [1] that $R\left(K^{ \pm} K^{ \pm}\right) \approx 3 \mathrm{fm}$. This is an important result for the study of signals of the Quark Gluon Plasma (QGP) (see refs. [2, 4, 8]). Moreover, we found that the long range correlations are strongly affected by Coulomb corrections (most probably because of the long range character of Coulomb interactions).

For the sake of reference, we show also in Table II and Fig. 5 results of our analysis of data for $\mathrm{S}+$ $\mathrm{Pb} \rightarrow \pi \pi+X$ reaction [6] (which were corrected by the Coulomb wave function method [9]) performed by using both eq. (7) and the "standard" formula (eq. (8)). As one can see there are no significant differences between parameters estimated by means of these two formulae, in particular the magnitude of the interaction region is in both cases almost the same.

Acknowledgements: The authors would like to thank S. Esumi, T. Nishimura, and S. D. Pandey (members of NA44 Collaboration) for useful conversations and correspondences. This work is partially supported by Japanese Grant-in-Aid for Scientific Research from the Ministry of Education, Science and Culture (\# 06640383).

\footnotetext{
${ }^{2}$ We have confirmed that the parameter $\epsilon$ depends on the magnitude of the interaction region $(R)$.

${ }^{3}$ This shows that Coulomb corrections can be important in the bigger range of momentum transfers than considered so far and justifies a posteriori our present investigation.
} 


\section{References}

[1] H. Beker et al. (NA44 Collaboration), Z. Phys. C 64 (1994) 209.

[2] M. Biyajima, T. Mizoguchi, Y. Nakata and G. Wilk, Prog. Theor. Phys. 92 (1994) 1223.

[3] M. G. Bowler, Phys. Lett. B270 (1991) 69.

[4] M. Biyajima and T. Mizoguchi, Coulomb wave function correction to Bose-Einstein correlations, preprint SULDP-94-9 (Dec. 1994).

[5] M. Biyajima, T. Mizoguchi, T. Osada and G. Wilk, Phys. Lett. B353 (1995) 340.

[6] H. Bøggild et al.(NA44 Collaboration), Phys. Lett. B302 (1993) 510.

[7] L. I. Schiff, Quantum Mechanics, 2nd Ed. (McGraw-Hill, New York, 1955), p. 117.

[8] S. Nagamiya, Nucl. Phys. A544 (1992) 5C.

[9] S. Pratt, T. Csörgö and J. Zimanyi, Phys. Rev. C42 (1990) 2646.

Table I: Parameters of kaonic $\mathrm{BEC}$ obtained from $\mathrm{S}+\mathrm{Pb}$ and $p+\mathrm{Pb}$ reactions.

\begin{tabular}{|c|c|c|c|c|c|c|}
\hline reaction & formula & $\mathrm{c}$ & $R[\mathrm{fm}]$ & $\lambda$ & $\gamma$ & $\chi^{2} / \mathrm{NDF}$ \\
\hline \multirow{4}{*}{$\begin{array}{l}\mathrm{S}+\mathrm{Pb} \rightarrow \\
K^{+} K^{+}+X\end{array}$} & \multirow{2}{*}{ standard } & $0.998 \pm 0.008$ & $3.384 \pm 0.171$ & $1.010 \pm 0.071$ & - & $58.0 / 33$ \\
\hline & & $1.101 \pm 0.022$ & $3.991 \pm 0.234$ & $0.977 \pm 0.078$ & $-0.420 \pm 0.079$ & $36.8 / 32$ \\
\hline & \multirow{2}{*}{ eq. (4) } & $0.966 \pm 0.007$ & $3.918 \pm 0.000$ & $0.804 \pm 0.059$ & - & $30.9 / 33$ \\
\hline & & $0.987 \pm 0.015$ & $4.285 \pm 0.000$ & $0.825 \pm 0.074$ & $-0.071 \pm 0.077$ & $30.9 / 32$ \\
\hline \multirow{4}{*}{$\begin{array}{l}\mathrm{S}+\mathrm{Pb} \rightarrow \\
K^{-} K^{-}+X\end{array}$} & \multirow{2}{*}{ standard } & $0.988 \pm 0.021$ & $3.058 \pm 0.285$ & $1.070 \pm 0.142$ & - & $31.9 / 32$ \\
\hline & & $0.989 \pm 0.066$ & $3.061 \pm 0.345$ & $1.069 \pm 0.159$ & $-0.004 \pm 0.279$ & $31.9 / 31$ \\
\hline & \multirow{2}{*}{ eq. (4) } & $0.944 \pm 0.017$ & $3.089 \pm 0.000$ & $0.836 \pm 0.111$ & - & $35.0 / 32$ \\
\hline & & $0.872 \pm 0.048$ & $3.066 \pm 0.012$ & $1.030 \pm 0.178$ & $0.411 \pm 0.277$ & $32.5 / 31$ \\
\hline \multirow{4}{*}{$\begin{array}{c}p+\mathrm{Pb} \rightarrow \\
K^{+} K^{+}+X\end{array}$} & \multirow{2}{*}{ standard } & $0.989 \pm 0.027$ & $2.134 \pm 0.291$ & $0.636 \pm 0.089$ & - & $34.3 / 26$ \\
\hline & & $1.149 \pm 0.078$ & $2.698 \pm 0.559$ & $0.493 \pm 0.114$ & $-0.546 \pm 0.237$ & $30.9 / 25$ \\
\hline & \multirow{2}{*}{ eq. (4) } & $0.975 \pm 0.019$ & $2.731 \pm 0.000$ & $0.520 \pm 0.091$ & - & $32.7 / 26$ \\
\hline & & $0.996 \pm 0.061$ & $2.310 \pm 0.001$ & $0.431 \pm 0.133$ & $-0.180 \pm 0.256$ & $30.7 / 25$ \\
\hline
\end{tabular}

\section{Figure Captions}

Fig. 1. (a) Analyses of data for $\mathrm{S}+\mathrm{Pb} \rightarrow K^{-} K^{-}+X$ reaction by eq. (四). (b) The same but with Coulomb corrections switched off for $q>q_{\text {limit }}=Q / 2$ limit. (Results for $K^{+} K^{+}$pair production 
Table II: Parameters of $\mathrm{S}+\mathrm{Pb} \rightarrow \pi^{ \pm} \pi^{ \pm}+X$ reactions.

\begin{tabular}{c|ccccc}
\hline \hline formula & $\mathrm{c}$ & $R[\mathrm{fm}]$ & $\lambda$ & $\gamma$ & $\chi^{2} / \mathrm{NDF}$ \\
\hline \multirow{2}{*}{ standard } & $0.800 \pm 0.003$ & $4.506 \pm 0.327$ & $0.461 \pm 0.042$ & - & $17.5 / 16$ \\
& $0.824 \pm 0.010$ & $5.015 \pm 0.421$ & $0.450 \pm 0.044$ & $-0.178 \pm 0.065$ & $10.9 / 15$ \\
\hline \multirow{2}{*}{ eq. (7) } & $0.800 \pm 0.003$ & $4.575 \pm 0.335$ & $0.509 \pm 0.048$ & - & $17.8 / 16$ \\
& $0.825 \pm 0.010$ & $5.105 \pm 0.436$ & $0.502 \pm 0.052$ & $-0.180 \pm 0.065$ & $11.0 / 15$ \\
\hline
\end{tabular}

looks similar with only change being in the value of $q_{\text {limit }}$ which depend on the value of radius parameter $R$.)

Fig. 2. Flow chart for our procedure of "seamless fitting (SF)".

Fig. 3. Results of $\mathrm{SF}$ for BEC for kaons produced in $\mathrm{S}+\mathrm{Pb}$ collisions; (a) for $K^{+} K^{+}$pairs; (b) for $K^{-} K^{-}$pairs.

Fig. 4. Results of SF for BEC for kaons produced in $p+\mathrm{Pb} \rightarrow K^{-} K^{-}+X$ reaction.

Fig. 5. Results of $\mathrm{SF}$ for $\mathrm{BEC}$ for pions produced in $\mathrm{S}+\mathrm{Pb} \rightarrow \pi^{+} \pi^{+}+X$ reaction. 
This figure "fig1-1.png" is available in "png" format from: http://arxiv.org/ps/hep-ph/9507293v2 
This figure "fig2-1.png" is available in "png" format from: http://arxiv.org/ps/hep-ph/9507293v2 
This figure "fig1-2.png" is available in "png" format from: http://arxiv.org/ps/hep-ph/9507293v2 